\title{
ON THE EXPONENT OF THE NK $_{0}$-GROUPS OF VIRTUALLY INFINITE CYCLIC GROUPS
}

\author{
FRANCIS X. CONNOLLY AND STRATOS PRASSIDIS ${ }^{1}$
}

\begin{abstract}
It is known that the $K$-theory of a large class of groups can be computed from the $K$ theory of their virtually infinite cyclic subgroups. On the other hand, Nil-groups appear to be the obstacle in calculations involving the $K$-theory of the latter. The main difficulty in the calculation of Nil-groups is that they are infinitely generated when they do not vanish. We develop methods for computing the exponent of $\mathrm{NK}_{0}$-groups that appear in the calculation of the $K_{0}$-groups of virtually infinite cyclic groups.
\end{abstract}

1991 Mathematics Subject Classification 18F25, 19A31

\section{INTRODUCTION}

The results of Farrell-Jones ([8]) have shown that the computation of the lower $K$-theory of a large class of groups depends on our ability to calculate the $K$-theory of virtually infinite cyclic groups. Virtually infinite cyclic groups are two ended groups and they are of two types:

- (Type (1)): Groups that admit an epimorphism, with kernel a finite group, to the infinite cyclic group $\mathbb{Z}$.

- (Type (2)): Groups that admit an epimorphism, with finite kernel, to the infinite dihedral group $D_{\infty}$.

The groups of the first type have the form $\Gamma=H \rtimes_{\alpha} \mathbb{Z}$ with $H$ a finite group. In this case, the twisted Bass-Heller-Swan formula can be used for the calculation of the $K$-theory of $\Gamma$. The Nilgroups that appear are the twisted versions of the classical Nil-groups introduced by Bass ([1], [7]). In the case that $\alpha$ is the identity, the exponents of the corresponding $\mathrm{NK}_{0}$-groups were computed in [4]. We will generalize their methods to the twisted $\mathrm{NK}_{0}-$ groups.

The algebraic structure of the groups in the second class is more complicated. A group $\Gamma$ in the second class has the form $\Gamma=G_{0} *_{H} G_{1}$ where the groups $G_{i}, i=0,1$, and $H$ are finite and $\left[G_{i}: H\right]=2$. In this case, the Nil-groups involved are Waldhausen's Nil-groups. We will use the special structure of $\Gamma$ to extend the methods of [10] and [11] to this case. Again, our methods heavily depend on the methods in [4].

Our interest in the exponent of the Nil-groups of virtually infinite cyclic groups arises from the fact that Nil-groups measure the difference between controlled and non-controlled phenomena. In many instances that difference is reflected on phenomena that are completely topological in nature ([5]). In a forthcoming paper ([6]) we will use the results on the exponent of the Nil-groups to compute the exponent of equivariant topological $K$-groups.

\footnotetext{
${ }^{1}$ Supported in part by a Research Grant from FAPERJ.
} 
We outline a construction for a positive integer $n$. Let $n=p_{1}^{k_{1}} p_{2}^{k_{2}} \ldots p_{s}^{k_{s}}$ be the decomposition of $n$ into prime numbers. Choose $\ell_{i}, i=1, \ldots s$, integers such that $p_{i}^{\ell_{i}} \geq k_{i} n$ for all $i$ and set $n^{\prime}=p_{1}^{\ell_{1}} p_{2}^{\ell_{2}} \ldots p_{s}^{\ell_{s}}$. The main result of the paper can be summarized as follows: Let $\Gamma=H \rtimes_{\alpha} \mathbb{Z}$ or $\Gamma=G_{0} *_{H} \Gamma_{1}$ where $H$ is a finite group of order $n$ and, in the second case, $\left[G_{0}: H\right]=\left[G_{1}: H\right]=2$. We call $H$ the base group of the virtually infinite cyclic group.

Theorem (Main Theorem). The $N K_{0}$-groups that appear in the splitting of the virtually infinite cyclic groups with base a finite group of order $n$ have exponent $n^{\prime}$.

The method of the proof follows closely the ideas developed in [4]. But the methods really go back to [3] and [9]. The main calculational tool is that for any finite group $H$ of order $n$ and an automorphism $\alpha: H \rightarrow H$, there is a pull-back diagram

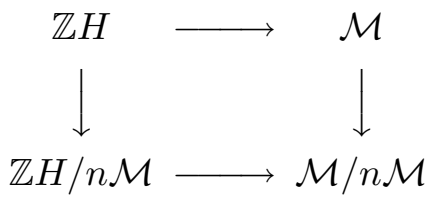

where $\mathcal{M}$ is a hereditary order such that

(1) $\mathbb{Z} H \subset \mathcal{M} \subset \mathbf{Q} H$.

(2) $\mathcal{M}$ is $\alpha$ invariant.

(3) $n \mathcal{M} \subset \mathbb{Z} H$.

For the $\mathrm{NK}_{0}$-groups of Type (1) groups, the calculations of [4] can be generalized in the twisted setting using the $\alpha$-invariant order $\mathcal{M}$. In the type (2) case, we use the description of the lower Waldhausen's Nil-groups as the reduced $K$-groups of an augmented ring ([11], [10]). The special structure of the groups in Type (2) allows us to express the augmented ring as a subring of the ring of the $2 \times 2$-matrices over a twisted polynomial ring. Methods similar to the ones in [4] can be applied to complete the proof in this case.

\section{Twisted NiL-GRoups}

This section is basically a modification of the argument given in [4] on the exponent of $\mathrm{NK}_{0^{-}}$ groups.

Let $H$ be a finite group of order $n$ and $\alpha: H \rightarrow H$ a group automorphism. We write $R=\mathbb{Z} H$. We denote by $\alpha$ the automorphism induced on $R$ by $\alpha$. Our objective is to calculate the exponent of the twisted Nil-group $\mathrm{NK}_{0}(R, \alpha)$. This group is defined as the kernel of the augmentation map

$$
\varepsilon: K_{0}\left(R_{\alpha}[t]\right) \rightarrow K_{0}(R) .
$$

The group $\mathrm{NK}_{0}(R, \alpha)$ appears in the twisted Bass-Heller-Swan formula given in [7] which calculates the K-theory of the twisted Laurent ring:

$$
K_{0}(R) \stackrel{1-\alpha_{*}}{\longrightarrow} K_{0}(R) \rightarrow K_{0}\left(R\left[t, t^{-1}\right]\right) / \mathcal{N} \rightarrow K_{-1}(R) \stackrel{1-\alpha_{*}}{\longrightarrow} K_{-1}(R)
$$

where $\mathcal{N}=\mathrm{NK}_{0}(R, \alpha) \oplus \mathrm{NK}_{0}\left(R, \alpha^{-1}\right)$. Actually the formula in [7] is given for $\mathrm{K}_{1}$-groups only but its extension to lower $K$-theory can be achieved using the techniques in [14], $§ 11$. 
Remark 2.1. In [7], Theorem 25, it is shown that if a $\operatorname{ring} R$ is regular and $\alpha$ is an automorphism of $R$, then the twisted polynomial and Laurent extensions of $R$ are regular rings. In particular, in this case all the NK-groups vanish ([13], p. 114).

The main tool for adjusting the proof in [4] to our case is the existence of a hereditary order $\mathcal{M}$ with the properties stated in the introduction. Another important ingredient is the twisted version of Proposition 2.12 in [1]. Recall that a ring $S$ is called quasi-regular if there is a two sided nilpotent ideal $J$ of $S$ such that $S / J$ is regular. We adjust this classical definition to our setting.

Definition 2.2. Let $R$ be a ring and $\alpha$ is an automorphism of $R$. Then $R$ is called $\alpha$-quasi-regular if there is a two sided nilpotent ideal which is $\alpha$ invariant such that $R / J$ is regular.

Remark 2.3. Let $R$ be an Artinian ring and $\alpha$ an automorphism of $R$. Then the Jacobson radical $J$ of $R$ is a two-sided nilpotent ideal and it is $\alpha$-invariant. Thus $R$ is $\alpha$-quasi-regular for each automorphism $\alpha$.

Lemma 2.4. Let $R$ be a ring and $\alpha: R \rightarrow R$ a ring automorphism. If $R$ is $\alpha$-quasi-regular then

$$
N K_{i}(R, \alpha)=0, \quad \text { for } i \leq 0 .
$$

Proof. We will show the result for $i=0$. The other cases follows from standard methods of lower $K$-theory. There is exists an $\alpha$-invariant two-sided ideal $J$ of $R$ such that $R / J$ is regular. Then the ring $(R / J)_{\alpha}[t]$ is also regular. Consider the commutative diagram induced by ring projections

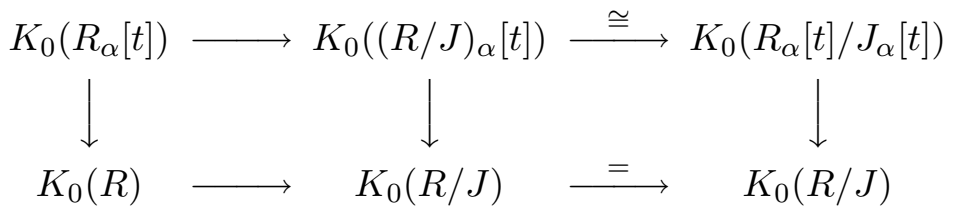

The composite horizontal maps are isomorphisms because $J_{\alpha}[t]$ and $J$ are nilpotent ideals. The middle vertical map is an isomorphism because $R / J$ is regular (Remark 2.1). Thus the first vertical map is also an isomorphism. The result follows.

We can now outline the argument given in [4]. The number $n^{\prime}$ is as in the introduction.

Theorem 2.5. With the above notation, the group $N K_{0}(R, \alpha)$ has exponent $n^{\prime}$.

Proof. Choose an $\alpha$-invariant hereditary order $\mathcal{M}$ as in the introduction. There are pull-back diagrams

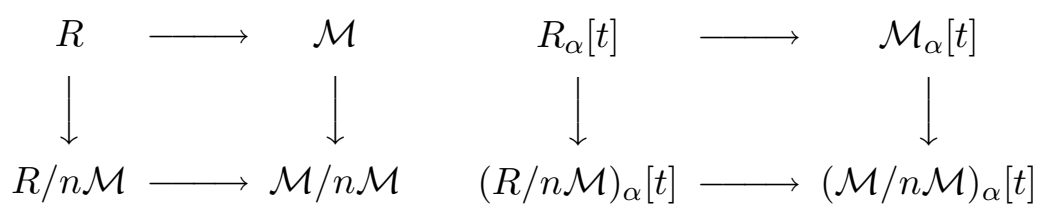

(we still denote by $\alpha$ the automorphism induced by $\alpha$ on all the rings involved) which induces a commutative diagram of exact sequences

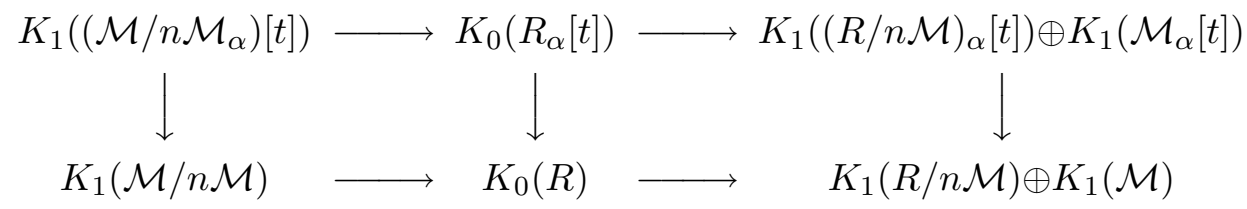


Taking kernels of the vertical maps results to an exact sequence

$$
\mathrm{NK}_{1}(\mathcal{M} / n \mathcal{M}, \alpha) \stackrel{\partial}{\rightarrow} \mathrm{NK}_{0}(R, \alpha) \rightarrow \mathrm{NK}_{0}(R / n \mathcal{M}, \alpha) \oplus \mathrm{NK}_{0}(\mathcal{M}, \alpha) .
$$

Since $\mathcal{M}$ is regular, $\operatorname{NK}_{0}(\mathcal{M}, \alpha)=0$. Since $R / n \mathcal{M}$ is finite, it is artinian. By Remark $2.3, R / n \mathcal{M}$ is $\alpha$-quasi-regular and by Lemma $2.4, \mathrm{NK}_{0}(R / n \mathcal{M}, \alpha)=0$. Thus the map $\partial$ is onto. So it is enough to compute the exponents of a set of generators of $\mathrm{NK}_{1}(\mathcal{M} / n \mathcal{M})$.

As in [4], Corollary 3.6, the map

$$
1+t J_{\alpha}[t] \rightarrow \mathrm{NK}_{1}(\mathcal{M}, \alpha)
$$

is onto. But the elements of the group $1+t J_{\alpha}[t]$ have exponent $n^{\prime}([4]$, p. 10). The result follows.

Remark 2.6. Using the arguments in [4], Proposition 3.7, the elements of the form $B=\left\{1+j t^{s}\right.$ : $j \in J, n \in \mathbb{N}\}$ generate the group $1+t J_{\alpha}[t]$. There is an action of the multiplicative monoid $\mathbb{N}$ on $B$ by $n .\left(1+j t^{s}\right)=1+j t^{s n}$, which induces an action on $1+t J_{\alpha}[t]$. Under this action $1+t J_{\alpha}[t]$ becomes a $\mathbb{Z}[\mathbb{N}]$-module with basis $\mathcal{B}=\{1+j t: j \in J\}$. That makes $\mathrm{NK}_{0}(R, \alpha)$ a finitely generated $\mathbb{Z}[\mathbb{N}]$-module.

\section{WAldhausen's NiL-Groups}

For the calculation of Waldhausen's Nil-groups we will use the interpretation given in [5], [10] and [11]. We will review briefly the construction. Let $\mathcal{T}$ be the category of triples $\mathbf{R}=\left(R ; B_{0}, B_{1}\right)$, where $B_{i}, i=0,1$ are two $R$-bimodules. A morphism in $\mathcal{T}$ is a triple

$$
\left(\phi, f_{0}, f_{1}\right):\left(R ; B_{0}, B_{1}\right) \rightarrow\left(S, C_{0}, C_{1}\right)
$$

where $\phi: R \rightarrow S$ is a ring homomorphism and $f_{i}: B_{i} \otimes_{R} S \rightarrow C_{i}, i=0,1$, are $R-S$-bimodule homomorphisms. There is a functor

$$
\rho: \mathcal{T} \rightarrow \text { Rings }, \quad \rho(\mathbf{R})=R_{\rho}=\left(\begin{array}{cc}
T_{R}\left(B_{1} \otimes_{R} B_{0}\right) & B_{1} \otimes_{R} T_{R}\left(B_{0} \otimes_{R} B_{1}\right) \\
B_{0} \otimes_{R} T_{R}\left(B_{1} \otimes_{R} B_{0}\right) & T_{R}\left(B_{0} \otimes_{R} B_{1}\right)
\end{array}\right)
$$

with multiplication given as matrix multiplication and on each entry by concatenation. The ring $R_{\rho}$ is constructed as follows: In [5], there is a construction of a twisted polynomial extension category associated to $\mathbf{R}$. The resulting category admits a basic object $b=(R, R)$ i.e. for each $v$ in the category there is an object $u$ such that $u \oplus v$ is isomorphic to $b^{k}\left([2]\right.$, p. 197). The ring $R_{\rho}$ is the endomorphism ring of the basic object in the twisted polynomial extension ([11], Proposition 2.3). The basic result is that the lower Waldhausen's Nil-groups associated to the triple $\mathbf{R}$ can be computed using the $K$-theory of the ring $R_{\rho}$. More specifically, there is a natural augmentation map

$$
R_{\rho} \stackrel{\varepsilon}{\longrightarrow}\left(\begin{array}{cc}
R & 0 \\
0 & R
\end{array}\right)
$$

We write $\mathrm{NK}_{i}(\mathbf{R})$ for the kernel of the map induced by $\varepsilon$ on $K_{i}$-groups. Then there is a natural isomorphism ([5], [11])

$$
\mathrm{NK}_{i}(\mathbf{R}) \cong \widetilde{\mathrm{Nil}}_{i-1}^{W}(\mathbf{R}), \quad \text { for } i \leq 1 .
$$

Thus vanishing results of Waldhausen's Nil-groups can be applied to the NK-groups. 
We now formulate the Nil-groups of interest. Let $\Gamma$ be a virtually infinite cyclic group of type (2). Then $\Gamma$ admits a decomposition as an amalgamated free product of finite groups

$$
\Gamma=G_{0} *_{H} G_{1}, \quad\left[G_{0}: H\right]=\left[G_{1}: H\right]=2 .
$$

Then the Waldhausen's Nil-groups are defined using the triple $\left(\mathbb{Z} H ; \mathbb{Z}\left[G_{0}-H\right], \mathbb{Z}\left[G_{1}-H\right]\right)$. Choosing right coset representatives, we see that the $\mathbb{Z} H$-bimodules $\mathbb{Z}\left[G_{i}-H\right], i=0,1$, are isomorphic to $\mathbb{Z} H$ as left $\mathbb{Z}$-modules, but the right action is twisted by an automorphism $\mathbb{Z} H$, induced by an automorphism of $H$.

We can now formulate the general setup. Let $R$ be a ring with one and $\alpha: R \rightarrow R$ a ring automorphism. We denote by $R^{\alpha}$ the $R-R$-bimodule which is $R$ as a left $R$-module but the right multiplication is given by: $x \cdot r=x \alpha(r)$. Our objective is to study the lower Waldhausen's Nil-groups of the form $\widetilde{\mathrm{Nil}_{*}} W\left(R ; R^{\alpha}, R^{\beta}\right)$ where $\alpha$ and $\beta$ are automorphisms of $R$. From now on, $\otimes$ means $\otimes_{R}$.

We start with a basic algebraic observation.

Lemma 3.1. With the above notation, there is an R-bimodule isomorphism

$$
i_{\alpha, \beta}: R^{\alpha} \otimes R^{\beta} \rightarrow R^{\alpha \beta}
$$

Proof. The isomorphism is given by

$$
i_{\alpha, \beta}(x \otimes y)=x \alpha(y)
$$

Proposition 3.2. There is an isomorphism

$$
\iota: \widetilde{N i l} l_{i}^{W}\left(R ; R^{\alpha}, R^{\beta}\right) \rightarrow \widetilde{N i l}_{i}^{W}\left(R ; R^{\alpha \beta}, R\right), \quad \text { for all } i \in \mathbb{Z} .
$$

Proof. We write $\mathbf{R}=\left(R ; R^{\alpha}, R^{\beta}\right)$. The Waldhausen's Nil-groups are defined using an exact category $\mathcal{N} i l(\mathbf{R})$ with objects quadruples $(P, Q ; p, q)$, where $P$ and $Q$ are finitely generated projective right $R$-modules and

$$
p: P \rightarrow Q \otimes R^{\alpha}, \quad q: Q \rightarrow P \otimes R^{\beta}
$$

is a pair of $R$-homomorphisms such that the compositions

$$
\begin{aligned}
& P \stackrel{p}{\longrightarrow} Q \otimes R^{\alpha} \stackrel{q \otimes 1}{\longrightarrow} P \otimes R^{\beta} \otimes R^{\alpha} \ldots \\
& Q \stackrel{q}{\longrightarrow} P \otimes R^{\beta} \stackrel{p \otimes 1}{\longrightarrow} P \otimes R^{\alpha} \otimes R^{\beta} \ldots
\end{aligned}
$$

are zero after finitely many steps. Morphisms are morphisms on the modules that are compatible with the maps. Exact sequences are defined the obvious way. Notice that there is a forgetful functor $\phi: \mathcal{N} i l(\mathbf{R}) \rightarrow \mathcal{P}_{R} \times \mathcal{P}_{R}$, where $\mathcal{P}_{R}$ is the category of finitely generated projective right $R$-modules. Then

$$
\widetilde{N i l}_{i}^{W}(\mathbf{R})=\operatorname{ker}\left(K_{i}(\mathcal{N} i l(\mathbf{R})) \stackrel{\phi_{i}}{\longrightarrow} K_{i}\left(\mathcal{P}_{R} \times \mathcal{P}_{R}\right)\right) .
$$

Set $\mathbf{R}^{\prime}=\left(R ; R^{\alpha \beta}, R\right)$. We will define an equivalence of categories

$$
\iota: \mathcal{N} i l(\mathbf{R}) \rightarrow \mathcal{N} i l\left(\mathbf{R}^{\prime}\right) .
$$


On objects, we define

$$
\iota(P, Q ; p, q)=\left(P \otimes R^{\beta}, Q ;\left(1_{Q} \otimes i_{\alpha, \beta}\right) \circ\left(p \otimes 1_{R^{\beta}}\right), q\right)
$$

(for every right $R$-module $M$ we will always identify $M \otimes R$ with $M$ in the natural way). If the sequence of maps, as in $\left(^{*}\right)$, starts with $P \otimes R^{\beta}$, then the resulting composition of maps is equal to the original composition tensored with the identity on $R^{\beta}$. If the sequence starts with $Q$ the resulting composition is equal to the original. In both cases it will be eventually zero. Thus the resulting quadruple is an object of $\mathcal{N} i l\left(\mathbf{R}^{\prime}\right)$. On morphisms $\iota$ is defined by

$$
\iota(f, g)=\left(f \otimes 1_{R^{\beta}}, g\right) .
$$

The functor $\iota$ is exact because $R^{\beta}$ is a free (and thus flat) left $R$-module.

We can now describe the inverse of $\iota$. That will be an exact functor

$$
\iota^{\prime}: \mathcal{N} i l\left(\mathbf{R}^{\prime}\right) \rightarrow \mathcal{N} i l(\mathbf{R}) .
$$

For a quadruple $\left(P^{\prime}, Q^{\prime} ; p^{\prime}, q^{\prime}\right)$ with

$$
p^{\prime}: P^{\prime} \rightarrow Q^{\prime} \otimes R^{\alpha \beta}, \quad q^{\prime}: Q^{\prime} \rightarrow P^{\prime}
$$

we define

$$
\iota^{\prime}\left(P^{\prime}, Q^{\prime} ; p^{\prime}, q^{\prime}\right)=\left(P^{\prime} \otimes R^{\beta^{-1}}, Q^{\prime} ;\left(1_{Q} \otimes i_{\alpha \beta, \beta^{-1}}\right) \circ\left(p^{\prime} \otimes 1_{R^{\beta^{-1}}}\right),\left(1_{P^{\prime}} \otimes i_{\beta^{-1}, \beta^{\circ}} q^{\prime}\right) .\right.
$$

For the same reasons as $\iota, \iota^{\prime}$ defines an exact functor on the $\mathcal{N} i l$-categories.

It is immediate to check that $\iota$ and $\iota^{\prime}$ define an equivalence between the two categories. Thus $\iota$ induces an isomorphism on the $K$-groups of the $\mathcal{N}$ il-categories. The result follows.

Corollary 3.3. There is an isomorphism

$$
N K_{i}\left(R ; R^{\alpha}, R^{\beta}\right) \cong N K_{i}\left(R ; R^{\alpha \beta}, R\right) \quad \text { for } i \leq 1 .
$$

Proof. The lower Waldhausen's Nil-groups and the lower NK-groups are isomorphic.

Remark 3.4. Actually the proof of Proposition 3.2 shows that there are isomorphisms for all $i \in \mathbb{Z}$ :

$$
\widetilde{\operatorname{Nil}}_{i}^{W}\left(R ; R^{\alpha}, R^{\beta}\right) \cong \widetilde{\operatorname{Nil}}_{i}^{W}\left(R ; R^{\alpha \beta}, R\right) \cong \widetilde{\operatorname{Nil}}_{i}^{W}\left(R ; R, R^{\alpha \beta}\right)
$$

Because of Corollary 3.3, we will concentrate on triples of the form $\mathbf{R}=\left(R ; R^{\alpha}, R\right)$ where $\alpha$ is an automorphism of $R$. In this case, we will first try to give a more manageable description of the ring $R_{\rho}$. If we set $B_{0}=R^{\alpha}, B_{1}=R$, then the ring $R_{\rho}$ takes the form

$$
R_{\rho}=\left(\begin{array}{cc}
T_{R}\left(R \otimes_{R} R^{\alpha}\right) & R \otimes_{R} T_{R}\left(R^{\alpha} \otimes_{R} R\right) \\
R^{\alpha} \otimes_{R} T_{R}\left(R \otimes_{R} R^{\alpha}\right) & T_{R}\left(R^{\alpha} \otimes_{R} R\right)
\end{array}\right)=\left(\begin{array}{cc}
T_{R}\left(R \otimes_{R} R^{\alpha}\right) & T_{R}\left(R \otimes_{R} R^{\alpha}\right) \otimes_{R} R \\
T_{R}\left(R^{\alpha} \otimes_{R} R\right) \otimes_{R} R^{\alpha} & T_{R}\left(R^{\alpha} \otimes_{R} R\right)
\end{array}\right)
$$

We will identify $R_{\rho}$ with a subring of the ring of $2 \times 2$-matrices over a twisted polynomial ring. The automorphism $\alpha$ induces an automorphism on the polynomial ring $R[s]$, denoted $\alpha$, with $\alpha(s)=s$. Let $R_{\alpha}^{(2)}[s, t]=(R[s])_{\alpha}[t]$. Then $R_{\alpha}^{(2)}[s, t]$ is the polynomial ring on two commuting variables which is isomorphic to $R[s, t]$ as a left $R$-module. The right action of $R$ on the variables $s$ and $t$ is given by:

$$
s . r=r s, \quad t . r=\alpha(r) t, \quad \text { for all } r \in R
$$


(the superscript ${ }^{(2)}$ in the notation suggests that the twist, by $\alpha$, occurs in the second variable). We will describe $R_{\rho}$ as a subring of $M_{2}\left(R_{\alpha}^{(2)}[s, t]\right)$. Set

$$
R_{\alpha, \mathrm{d}}^{(2)}[s, t]=\left\{p \in R_{\alpha}^{(2)}[s, t]: \operatorname{degree}_{s}(p)=\operatorname{degree}_{t}(p)\right\}
$$

for the subring of $R_{\alpha}^{(2)}[s, t]$ generated by all the monomials which have equal degrees with respect to the variables.

Proposition 3.5. There is a ring isomorphism

$$
\mu: R_{\rho} \rightarrow R_{\rho}^{\prime}=\left(\begin{array}{cc}
R_{\alpha, d}^{(2)}[s, t] & R_{\alpha, d}^{(2)}[s, t] s \\
R_{\alpha, d}^{(2)}[s, t] t & R_{\alpha, d}^{(2)}[s, t]
\end{array}\right)
$$

Proof. We describe how to define $\mu$ on each entry of $R_{\rho}$. It is enough to show how to define $\mu$ on each component of an entry of $R_{\rho}$. We write 1 for the identity element of $R$ and $1_{\alpha}$ for the generator of $R_{\alpha}$ corresponding to 1 . We map 1 to $s, 1_{\alpha}$ to $t$ and tensor products of 1 and $1_{\alpha}$ to corresponding products of $s$ and $t$. Now every element in a component of an entry of $R_{\rho}$ can be represented in the form $r p$, where $r \in R$ and $p$ is a tensor product of 1's and $1_{\alpha}$ 's. We define $\mu(r p)=r \mu(p)$. We will show that $\mu$ is a ring homomorphism. We write $\left(1 \otimes 1_{\alpha}\right)^{\kappa}\left(1_{\alpha} \otimes 1\right)$ for the tensor product of $\kappa$-copies of $1 \otimes 1_{\alpha}\left(1_{\alpha} \otimes 1\right)$. We will check only that $\mu$ is a ring homomorphism on monomials. Actually we will check the first entry of the matrices. The general case follows similarly. Let $a$ and $b$ be two elements in $R_{\rho}$. Then $a$ and $b$ have the form

$$
\begin{aligned}
a & =\left(\begin{array}{cc}
r_{1,1}\left(1 \otimes 1_{\alpha}\right)^{\kappa_{1,1}} & r_{1,2}\left(1 \otimes 1_{\alpha}\right)^{\kappa_{1,2} \otimes 1} \\
r_{2,1}\left(1_{\alpha} \otimes 1\right)^{\kappa_{2,1}} \otimes 1_{\alpha} & r_{2,2}\left(1_{\alpha} \otimes 1\right)^{\kappa_{2,2}}
\end{array}\right), \\
b & =\left(\begin{array}{cc}
r_{1,1}^{\prime}\left(1 \otimes 1_{\alpha}\right)^{\kappa_{1,1}^{\prime}} & r_{1,2}^{\prime}\left(1 \otimes 1_{\alpha}\right)^{\kappa_{1,2}^{\prime} \otimes 1} \\
r_{2,1}^{\prime}\left(1_{\alpha} \otimes 1\right)^{\kappa_{2,1}^{\prime} \otimes 1_{\alpha}} & r_{2,2}^{\prime}\left(1_{\alpha} \otimes 1\right)^{\kappa_{2,2}^{\prime}}
\end{array}\right)
\end{aligned}
$$

Their images are

$$
\begin{aligned}
& \mu(a)=\left(\begin{array}{cc}
r_{1,1}(s t)^{\kappa_{1,1}} & r_{1,2} s^{\kappa_{1,2}+1} t^{\kappa_{1,2}} \\
r_{2,1} s^{\kappa_{2,1}} t^{\kappa_{2,1}+1} & r_{2,2}(s t)^{\kappa_{2,2}}
\end{array}\right) \\
& \mu(b)=\left(\begin{array}{cc}
r_{1,1}^{\prime}(s t)^{\kappa_{1,1}^{\prime}} & r_{1,2}^{\prime} s^{\kappa_{1,2}^{\prime}+1} t^{\kappa_{1,2}} \\
r_{2,1}^{\prime} s^{\kappa_{2,1}^{\prime}} t^{\kappa_{2,1}^{\prime}+1} & r_{2,2}^{\prime}(s t)^{\kappa_{2,2}^{\prime}}
\end{array}\right)
\end{aligned}
$$

We compute the entry at $(1,1)$ of the product $a b$ in $R_{\rho}$.

$$
\begin{aligned}
p_{1,1}=\left[r_{1,1}\left(1 \otimes 1_{\alpha}\right)^{\kappa_{1,1}}\right]\left[r_{1,1}^{\prime}\left(1 \otimes 1_{\alpha}\right)^{\kappa_{1,1}^{\prime}}\right] & +\left[r_{1,2}\left(1 \otimes 1_{\alpha}\right)^{\kappa_{1,2}} \otimes 1\right]\left[r_{2,1}^{\prime}\left(1_{\alpha} \otimes 1\right)^{\kappa_{2,1}^{\prime}} \otimes 1_{\alpha}\right]= \\
r_{1,1} \alpha^{\kappa_{1,1}}\left(r_{1,1}^{\prime}\right)\left(1 \otimes 1_{\alpha}\right)^{\kappa_{1,1}+\kappa_{1,1}^{\prime}} & +r_{1,2} \alpha^{\kappa_{1,2}}\left(r_{2,1}^{\prime}\right)\left(1 \otimes 1_{\alpha}\right)^{\kappa_{1,2}+\kappa_{2,1}^{\prime}+1}
\end{aligned}
$$

Thus the $(1,1)$ entry of $\mu(a b)$ will be

$$
\mu\left(p_{1,1}\right)=r_{1,2} \alpha^{\kappa_{1,2}}\left(r_{2,1}^{\prime}\right)(s t)^{\kappa_{1,1}+\kappa_{1,1}^{\prime}}+r_{1,2} \alpha^{\kappa_{1,2}}\left(r_{2,1}^{\prime}\right)(s t)^{\kappa_{1,2}+\kappa_{2,1}^{\prime}+1} .
$$


Now we compute the $(1,1)$-entry of $\mu(a) \mu(b)$ :

$$
\begin{aligned}
& r_{1,1}(s t)^{\kappa_{1,1}} r_{1,1}^{\prime}(s t)^{\kappa_{1,1}^{\prime}}+r_{1,2} s^{\kappa_{1,2}+1} t^{\kappa_{1,2}} r_{2,1}^{\prime} s^{\kappa_{2,1}^{\prime}} t^{\kappa_{2,1}^{\prime}+1}= \\
& r_{1,2} \alpha^{\kappa_{1,2}}\left(r_{2,1}^{\prime}\right)(s t)^{\kappa_{1,1}+\kappa_{1,1}^{\prime}}+r_{1,2} \alpha^{\kappa_{1,2}}\left(r_{2,1}^{\prime}\right)(s t)^{\kappa_{1,2}+\kappa_{2,1}^{\prime}+1}=\mu\left(p_{1,1}\right) .
\end{aligned}
$$

The inverse of $\mu$ is constructed in the obvious way.

There is a natural augmentation map

$$
\varepsilon^{\prime}: R_{\rho}^{\prime} \rightarrow\left(\begin{array}{cc}
R & 0 \\
0 & R
\end{array}\right)
$$

such that $\mu \circ \varepsilon=\varepsilon^{\prime}$ where $\varepsilon$ is the corresponding augmentation map on $R_{\rho}$. The following result is a consequence of Proposition 3.5.

Corollary 3.6. The groups $N K_{i}(\mathbf{R}), i \leq 1$, are isomorphic to the kernel of the map induced by the augmentation $\varepsilon^{\prime}$ :

$$
N K_{i}(\mathbf{R})=\operatorname{ker}\left(K_{i}\left(R_{\rho}^{\prime}\right) \stackrel{\varepsilon_{i}^{\prime}}{\longrightarrow} K_{i}(R \times R)\right), \quad i \leq 1 .
$$

Proposition 3.7. If $R$ is regular then $N K_{i}\left(R ; R^{\alpha}, R\right)=0$ for all $i \leq 1$. In particular, the augmentation maps $\varepsilon_{i}$ and $\varepsilon_{i}^{\prime}$ induce an isomorphisms on lower $K$-groups.

Proof. For $i \leq 1$, the groups $\operatorname{NK}_{i}\left(R ; R^{\alpha}, R\right)$ are isomorphic to Waldhausen's groups $\widetilde{N i l}_{i-1}^{W}\left(R ; R^{\alpha}, R\right)$ which vanish for $R$ regular ([15], [16]). The result follows.

Let $J$ be a two-sided ideal of $R$ which is invariant under the automorphism $\alpha$. Then the projection $R \rightarrow R / J$ induces a morphism of triples

$$
\left(R ; R^{\alpha}, R\right) \rightarrow\left(R / J ;(R / J)^{\alpha}, R / J\right)
$$

and thus a ring homomorphism

$$
\chi_{J}: R_{\rho}^{\prime} \rightarrow(R / J)_{\rho}^{\prime} .
$$

Lemma 3.8. Using the above notation,

$$
(R / J)_{\rho}^{\prime} \cong R_{\rho}^{\prime} / J_{\rho}^{\prime}
$$

where $J_{\rho}^{\prime}$ is the two sided ideal of $R_{\rho}^{\prime}$ generated by $J \times J$ i.e.

$$
J_{\rho}^{\prime}=\left\langle\left(\begin{array}{ll}
J & 0 \\
0 & J
\end{array}\right)\right\rangle
$$

Proof. The proof is the same as in [11], Proposition 2.10.

We also denote by $\chi_{J}: R_{\rho}^{\prime} \rightarrow R_{\rho}^{\prime} / J_{\rho}^{\prime}$ the natural projection homomorphism.

Remark 3.9. With the setting as in Lemma 3.8,

$$
J_{\rho}^{\prime}=\left(\begin{array}{cc}
J_{\alpha, \mathrm{d}}^{(2)}[s, t] & J_{\alpha, \mathrm{d}}^{(2)}[s, t] s \\
J_{\alpha, \mathrm{d}}^{(2)}[s, t] t & J_{\alpha, \mathrm{d}}^{(2)}[s, t]
\end{array}\right)
$$

In particular, if $J$ is nilpotent $J_{\rho}^{\prime}$ is also nilpotent. 
We are interested in determining the properties of the map that is induced in $K$-theory by $\chi_{J}$.

Proposition 3.10. Let $J$ be a nilpotent two-sided ideal of $R$ which is invariant under $\alpha$. Then the ring homomorphism $\chi_{J}$, described above, induces an isomorphism in $K_{i}$-groups, for $i \leq 0$.

Proof. Using Lemma 3.8, the map $\chi_{J}$ can be described as a quotient map

$$
\chi_{J}: R_{\rho}^{\prime} \rightarrow R_{\rho}^{\prime} / J_{\rho}^{\prime} .
$$

But the ideal $J_{\rho}^{\prime}$ is a nilpotent ideal of $R_{\rho}^{\prime}$ (Remark 3.9). The result follows from [1], Proposition 2.12 (also [11], Proposition 2.11).

Corollary 3.11. Let $R$ be an Artinian ring and $J$ its Jacobson radical. Then the quotient map $\chi_{J}$ induces isomorphism on $K_{i}$-groups for $i \leq 0$.

Proof. Since $R$ is Artinian, $J$ is nilpotent. Also, $J$ is invariant of every automorphism of $R$. The result follows from Proposition 3.10.

Corollary 3.12. Let $R$ be an $\alpha$-quasi-regular ring. Then $N K_{i}\left(R ; R^{\alpha}, R\right)=0$, for all $i \leq 0$.

Proof. Let $J$ be a two-sided, $\alpha$-invariant nilpotent ideal of $R$ such that the ring $R / J$ is regular. By Proposition 3.10, the map

$$
\left(\chi_{J}\right)_{i}: K_{i}\left(R_{\rho}^{\prime}\right) \rightarrow K_{i}\left((R / J)_{\rho}^{\prime}\right)
$$

is an isomorphism for $i \leq 0$. There is a commutative diagram

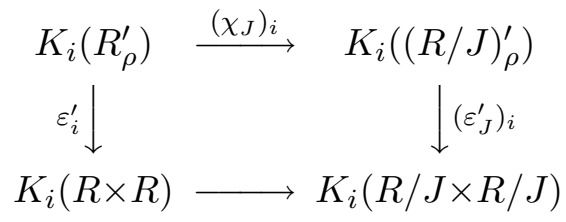

where the vertical maps are induced by the augmentations. Since $R / J$ is regular, $\varepsilon_{J}^{\prime}$ is an isomorphism (Proposition 3.7). Also, since $J$ is nilpotent the bottom map is an isomorphism. Thus $\varepsilon^{\prime}$ is an isomorphism and $\mathrm{NK}_{i}\left(R ; R^{\alpha}, R\right)=0$ for $i \leq 0$.

Now we specialize to the case that $R=\mathbb{Z} H$, the group ring of a finite group $H$ of order $n$. Let $\alpha$ be an automorphism of $R$ induced by an automorphism of $H$. Choose a hereditary order $\mathcal{M}$ as in the introduction Then we can define triples in $\mathcal{T}$,

$$
\begin{aligned}
\mathbf{M} & =\left(\mathcal{M} ; \mathcal{M}^{\alpha}, \mathcal{M}\right) \\
\mathbf{R} / n \mathbf{M} & =\left(R / n \mathcal{M} ;(R / n \mathcal{M})^{\alpha}, R / n \mathcal{M}\right), \\
\mathbf{M} / n \mathbf{M} & =\left(\mathcal{M} / n \mathcal{M} ;(\mathcal{M} / n \mathcal{M})^{\alpha}, \mathcal{M} / n \mathcal{M}\right)
\end{aligned}
$$

(we still use $\alpha$ to denote the homomorphism induced by $\alpha$ on the other rings involved in the calculation). The triples determine matrix rings (Proposition 3.5):

$$
\begin{aligned}
\mathcal{M}_{\rho}^{\prime} & \cong \mathcal{M}_{\rho} & & \text { corresponds to }\left(\mathcal{M} ; \mathcal{M}^{\alpha}, \mathcal{M}\right) \\
(R / n \mathcal{M})_{\rho}^{\prime} & \cong(R / n \mathcal{M})_{\rho} & & \text { corresponds to }\left(R / n \mathcal{M} ;(R / n \mathcal{M})^{\alpha}, R / n \mathcal{M}\right) \\
(\mathcal{M} / n \mathcal{M})_{\rho}^{\prime} & \cong(\mathcal{M} / n \mathcal{M})_{\rho} & & \text { corresponds to }\left(\mathcal{M} / n \mathcal{M} ;(\mathcal{M} / n \mathcal{M})^{\alpha}, \mathcal{M} / n \mathcal{M}\right)
\end{aligned}
$$


There is a commutative diagram of matrix rings

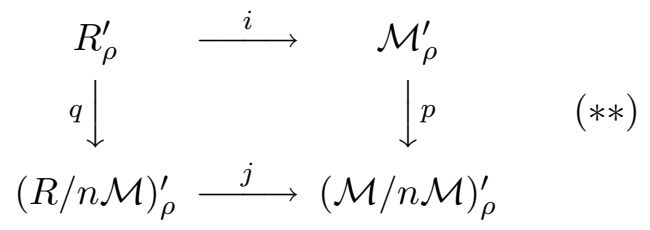

were $i$ and $j$ are induced by the inclusions and $p$ and $q$ by the projections. Actually, the maps in the diagram are induced by the maps of triples but this is not of use to us.

Proposition 3.13. The diagram (**) is a pull-back square of rings.

Proof. By construction, the sequence

$$
0 \rightarrow R_{\rho}^{\prime} \stackrel{(i,-q)}{\longrightarrow} \mathcal{M}_{\rho}^{\prime} \oplus(R / n \mathcal{M})_{\rho}^{\prime} \stackrel{(p \quad j)}{\longrightarrow}(\mathcal{M} / n \mathcal{M})_{\rho}^{\prime} \rightarrow 0
$$

is exact sequence of $R_{\rho}^{\prime}$-bimodules because it is exact on each entry of the matrix representation of the rings. The result follows.

Remark 3.14. There are also the classical pull-back diagrams

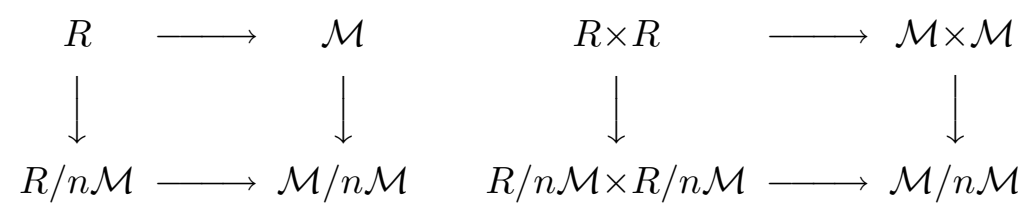

The result of Proposition 3.13 allows us to use Mayer-Vietoris type exact sequences for the calculation of the Nil-groups.

Proposition 3.15. There is an epimorphism

$$
\partial: N K_{1}\left(\mathcal{M} / n \mathcal{M} ;(\mathcal{M} / n \mathcal{M})^{\alpha}, \mathcal{M} / n \mathcal{M}\right) \rightarrow N K_{0}\left(R ; R^{\alpha}, R\right) .
$$

Proof. Combining the Mayer-Vietoris sequences for the diagram (**) and the second diagram in Remark 3.14, we obtain a commutative diagram of exact sequences

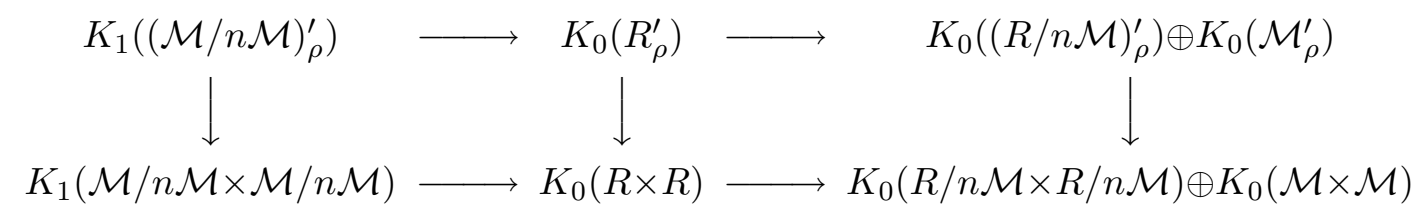

where the vertical maps are introduced by the augmentations. Using Corollary 3.6, we see that taking the kernels of the vertical maps induces an exact sequence

$$
\mathrm{NK}_{1}(\mathbf{M}) \stackrel{\partial}{\rightarrow} \mathrm{NK}_{0}(\mathbf{R}) \rightarrow \mathrm{NK}_{0}(\mathbf{R} / n \mathbf{M}) \oplus \mathrm{NK}_{0}(\mathbf{M})
$$

But the ring $R / n M$ is Artinian and thus $\alpha$-quasi-regular (Remark 2.3, Corollary 3.12) and $\mathcal{M}$ is regular. Thus the two NK-groups at the right end of the sequence vanish. The result follows.

The rest of the calculation of the exponent of the Nil-groups follows closely the methods used in [4] and [11]. We will outline the method of the proof. Remember that $R=\mathbb{Z} H$ and the order of the finite group $H$ is $n$, and $n^{\prime}$ is as in the introduction. 
Theorem 3.16. The group $N K_{0}\left(R ; R^{\alpha}, R\right)$ has exponent $n^{\prime}$ i.e.

$$
n^{\prime} N K_{0}\left(R ; R^{\alpha}, R\right)=0 .
$$

Proof. By Proposition 3.15, we need to calculate the exponent of the generators of the group $\mathrm{NK}_{1}\left(\mathcal{M} / n \mathcal{M} ;(\mathcal{M} / n \mathcal{M})^{\alpha}, \mathcal{M} / n \mathcal{M}\right)$. The method of proof of Proposition 4.9 in [11] applies here because $J_{\rho}^{\prime}$ is a nilpotent ideal (Remark 3.9). Let $I_{\rho}$ be the augmentation ideal

$$
I_{\rho}=\operatorname{ker}\left(\varepsilon^{\prime}:(\mathcal{M} / n \mathcal{M})_{\rho}^{\prime} \rightarrow \mathcal{M} / n \mathcal{M} \times \mathcal{M} / n \mathcal{M}\right) .
$$

Let $\widetilde{J}_{\rho}=J_{\rho}^{\prime} \cap I_{\rho}$. Then in Proposition 4.9 in [11], it was shown that there is an epimorphism

$$
1+\widetilde{J}_{\rho} \rightarrow \mathrm{NK}_{1}(\mathbf{M} / n \mathbf{M}) .
$$

Thus a set of generators of $\mathrm{NK}_{1}(\mathbf{M} / n \mathbf{M})$ can be defined by the image of a set of generators of $1+\widetilde{J}_{\rho}$. Again, interpreting the result in Proposition 4.9 in [11], a set of generators of $1+\widetilde{J}_{\rho}$ is given by element of the form

$$
\left(\begin{array}{cc}
1+p_{1,1} & 0 \\
0 & 1+p_{2,2}
\end{array}\right), \quad\left(\begin{array}{cc}
1 & p_{1,2} \\
0 & 1
\end{array}\right), \quad\left(\begin{array}{cc}
1 & 0 \\
p_{2,1} & 1
\end{array}\right)
$$

where $p_{1,1}$ and $p_{2,2}$ are in $J_{\alpha, \mathrm{d}}^{(2)}[s, t], p_{1,2} \in J_{\alpha, \mathrm{d}}^{(2)}[s, t] s$, and $p_{2,1} \in J_{\alpha, \mathrm{d}}^{(2)}[s, t] t$. The exponents of these elements is $n^{\prime}$ ([11], Theorem 5.1).

Then it is immediate that the same result applies to Waldhausen's Nil-groups.

Theorem 3.17. The Waldhausen's NK-groups that appear in the decomposition of a virtually infinite cyclic group with base $H$ of order $n$ have exponent $n^{\prime}$.

\section{REFERENCES}

[1] H. Bass, Algebraic K-theory, Benjamin (1968).

[2] H. Bass, Unitary algebraic K-theory, Proceedings of the Conference in Algebraic K-theory, Battelle 1972, Springer Lecture Notes in Mathematics v. 343, 57 - 265 (1973).

[3] D. W. Carter, Localization in lower K-theory, Comm. Algebra 8, 603-622 (1980).

[4] F. X. Connolly and M. O. M. daSilva, The groups $N^{r} K_{0}(\mathbb{Z} \pi)$ are finitely generated $\mathbb{Z}\left[\mathbb{N}^{r}\right]$-modules if $\pi$ is a finite group, K-theory 9, 1 - 11 (1995).

[5] F. X. Connolly and T. Koźniewski, Nil-groups in K-theory and surgery theory, Forum Math. 7, 45-76 (1995).

[6] F. X. Connolly, S. Prassidis, On the exponent of the equivariant topological K-groups, preprint.

[7] F. T. Farrell and W. C. Hsiang, A formula for $K_{1} R_{\alpha}[T]$, Proc. Symp. Pure Math. vol. 17, Applications of Categorical Algebra, Amer. Math. Soc., 192-218, (1970).

[8] F. T. Farrell and J. E. Jones, Isomorphism conjectures in algebraic K-theory, J. of AMS 6, 249-298 (1993).

[9] F. T. Farrell and J. E. Jones, The lower algebraic K-theory of virtually infinite cyclic groups, K-theory 9, 13-30 (1995).

[10] H. J. Munkholm and S. Prassidis, On the vanishing of certain K-theory Nil-groups, Proceedings of BCAT 1998, Barcelona 1998 (to appear).

[11] D.J.Pineda and S. Prassidis, On the lower Waldhausen's Nil-groups, Forum Math. (to appear).

[12] S. Prassidis, A split exact sequence of equivariant K-groups of virtually nilpotent groups, K-theory 11, $397-415$ (1997).

[13] D. Quillen, Higher algebraic K-theory, Lecture Notes in Math., vol 341, Springer-Verlag, 1973, 85-147. 
[14] A. Ranicki, Lower K- and L-Theory, Cambridge University Press, Cambridge 1992.

[15] F. Waldhausen, Whitehead groups of generalized free products, Proceedings of the Conference in Algebraic Ktheory, Battelle 1972, Springer Lecture Notes in Mathematics v. 342 (1973).

[16] F. Waldhausen, Algebraic K-theory of generalized free products, Ann. of Math. 108, 135 - 256 (1978).

Department of Mathematics, University of Notre Dame, Notre Dame IN 46556, U.S.A.

E-mail address: connolly.1.@nd.edu

CoordenaÇão de Pós GraduaÇão em Matemática, Rua Mário Santos Braga, $s / n^{\circ}-7^{\circ} \underline{o}$ Andar

VAlonguinho, Niterói, RJ 24020-005, BraZiL

E-mail address: stratos@mat.uff.br 